\title{
Exploring the Feasibility of Indoor Human Positioning by Using Radio Channel Properties of a Single Link
}

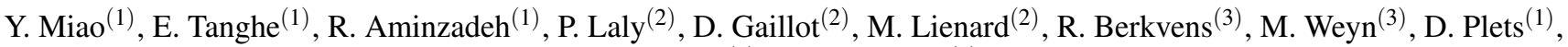 \\ L. Martens ${ }^{(1)}$ and W. Joseph ${ }^{(1)}$ \\ (1) Ghent University - IMEC, Technologiepark-Zwijnaarde 15, 9052 Gent, Belgium \\ (2) Lille University - TELICE, Batiment P3, F-59655, Villeneuve d'Ascq, Lille, France
}

(3) University of Antwerp - IMEC-IDLab, Groenenborgerlaan 171, 2020 Antwerp, Belgium

\begin{abstract}
This paper explores the feasibility of positioning human by using a single link of multiple-input multiple-output radio channel without ultra-wide bandwidth. While the background channel is measured without the presence of person, the current channel is measured with person(s) standing at several locations in a meeting room facing either in parallel or in orthogonal to the direct line between transmit and receive antennas. The background and current channels are compared by means of the power delay profile, the power angular spectrum, and the estimated plane wave paths. It is found that both the detection and the positioning of person(s) are feasible using the variations of radio channel properties, but extra algorithm and specific deployment are needed for positioning accurately.
\end{abstract}

\section{Introduction}

Device-free passive human localization has been a hot topic for emerging assisted-living applications. A device-free localization (DFL) system does not require human to wear or carry any device to be detected or localized. Radio-based DFL system, comparing to the camera- or thermal-based DFL systems, does not have constraints on visibility conditions of physical environment. In addition, radio-based DFL system is easily compatible with existing wireless network in buildings. Benefit from the multipath richness of indoor radio propagation channel, it is interesting to localize target human by observing the changes caused by the interaction between radio waves and target [1]. Those changes could be the time of arrival, the angle of departure, the angle of arrival, or the signal strength of the specific type of propagation mechanism [2,3]. The "break-in" person may cause new radio wave paths, may leads the existing paths to change, or may not influence some path at all [1].

To explore the feasibility of human detection and positioning for in-room scenario by using the radio propagation channel characteristics of a single link, this paper compares the delay power profile (PDP), the power angular spectrum (PAS) and the estimated plane wave paths from the channels with and without the presence of human. The channels were measured in a meeting room by MIMOSA channel sounder [4] with patch arrays at link ends without ultrawide bandwidth. It is found that 1) the detection of human can utilize variations on PDP (say, the first peak level, the reverberation slope) as well as PAS, 2) the positioning of human can utilize PAS and the extracted paths, but extra algorithm is needed since the variations appear at locations that may not be directly linked to the exact human position.

\section{Measurement Campaign}

Radio channel measurements were performed with the MIMOSA channel sounder [4]. The transmission bandwidth is $80 \mathrm{MHz}$ and the center frequency is $1.35 \mathrm{GHz}$. The sounder supports 8 transmitting and 16 receiving parallel channels. Orthogonal frequency division multiplexing (OFDM) is used to encode the digital transmit symbols, where $80 \mathrm{MHz}$ bandwidth is divided into 6560 sub-carriers and further equally divided among 8 parallel transmit channels. The inter-frequency spacing is hence $12.2 \mathrm{kHz}$ and an OFDM symbol duration is $82 \mu \mathrm{s}$. The cyclic prefix is chosen to be a fraction of the symbol duration. Each transmit channel is connected to a two-state RF switch, hence $16 \times 16$ channels can be measured and costs at most 328 $\mu$ s for one snapshot. At link ends, planar arrays with 8 dual-polarized patch antennas are deployed. As is shown in Fig. 1, with the array vertically placed in the indicated global coordinate, the radiation pattern in the cut planes are as in Fig. 2 where $\theta$ and $\phi$ are the co-elevation and azimuth angles. The radiation pattern of each array element is calculated by rotation and translation of simulated patch pattern.

The measurements were conducted in a meeting room in Lille University, France. Fig. 3 shows the floor plan with $\mathrm{Tx}$ and Rx locations. The floor and ceiling are made of concrete, the outer wall (top side in Fig. 3) consists of windows and concrete structures, and the inner walls are plasterboard. In addition, there are meeting tables and chairs. $\mathrm{Tx}$ and Rx arrays were set to fixed locations. They were parallel to wall surfaces and radiated radio waves towards inside room. The channels were measured with and without person(s), where the former considered up to 4 persons standing at different 6 locations and facing either in 


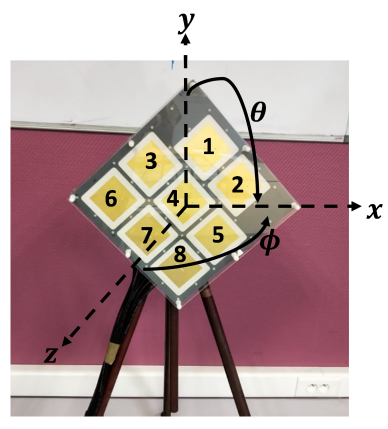

Figure 1. Dual-polarized patch antenna array
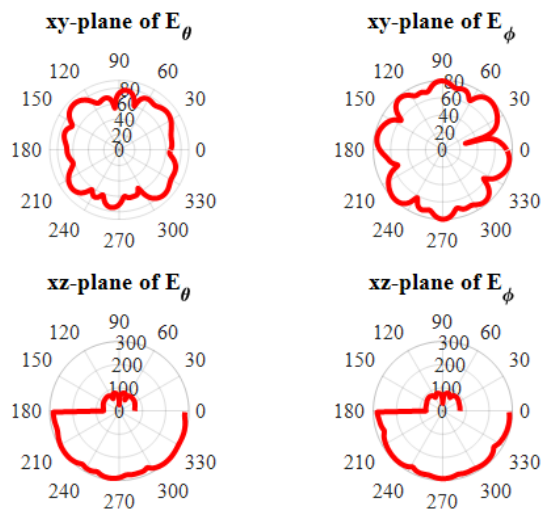

Figure 2. Patch array radiation pattern in different planes

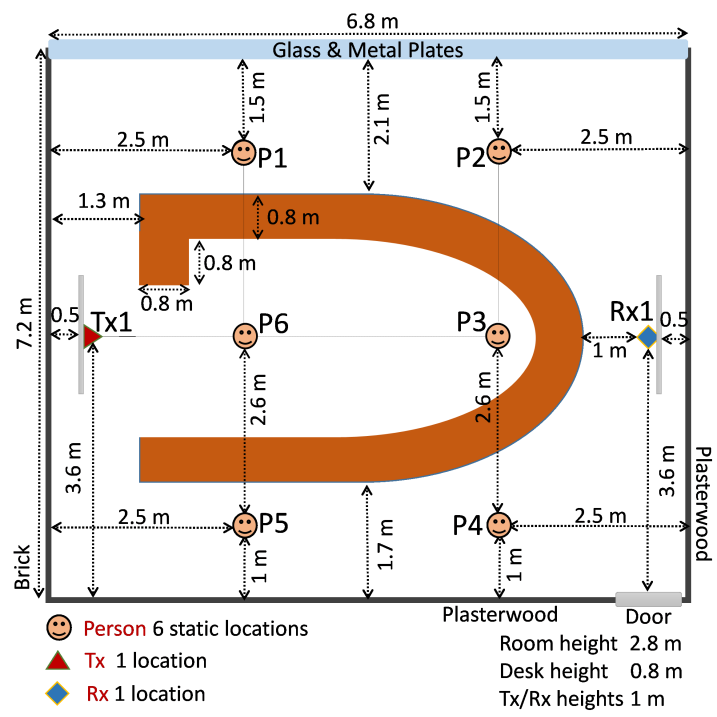

Figure 3. Floor plan with $\mathrm{Tx}, \mathrm{Rx}$, person locations

parallel (FP) or orthogonal (FO) to the direct line between $\mathrm{Tx} / \mathrm{Rx}$. The measurement scenarios are summarized in Table 1, where (Y,E,F,N) stand for different persons. While $\mathrm{Y}$ is an average-sized woman, E, F are average-sized men and $\mathrm{N}$ is a slim man. Each radio channel was measured 15 times (snapshots) assuming static condition.
Table 1. Measurement Scenarios

\begin{tabular}{ll}
\hline Without person & \\
\hline \multirow{2}{*}{ With 1 person } & Y FO/FP @ P1,P2,P3,P4,P5,P6 \\
& E FO/FP @ P1,P2,P3,P4,P5,P6 \\
\hline & (Y,E) (FO,FO)/(FP,FP) @ P1P2, P1P3, \\
& P1P4, P1P5, P1P6, P2P1, P2P3, P2P4, \\
With 2 persons & P2P5, P2P6, P3P1, P3P2, P3P4, P3P5, \\
& P3P6, P4P1, P4P2, P4P3, P4P5, P4P6, \\
& P5P1, P5P2, P5P3, P5P4, P5P6, P6P1, \\
& P6P2, P6P3, P6P4, P6P5 \\
\hline \multirow{3}{*}{ With 3 persons } & (Y,E,F) (FO,FO,FO)/(FP,FP,FP) @ P1P5P4, \\
& P1P6P3, P3P4P2, P5P1P2, P5P6P3, \\
& P6P1P2, P6P5P1, P6P5P4 \\
\hline \multirow{3}{*}{ With 4 persons } & (Y,E,F,N) (FO,FO,FO,FO)/(FP,FP,FP,FP) \\
& @ P3P1P2P6, P3P5P4P6, P5P1P2P3, \\
& P5P1P2P4, P5P6P3P2, P6P5P4P2
\end{tabular}

\section{Radio Channel Properties}

The PDP of channel transfer function (CTF) $\boldsymbol{H}_{X Y}$ is

$$
\operatorname{PDP}_{X Y}=\sum_{n_{\mathrm{r}}=1}^{8} \sum_{n_{\mathrm{t}}=1}^{8} \mid\left.\operatorname{IFFT}\left\{\text { HannWindow }\left\{\boldsymbol{H}_{X Y, n_{\mathrm{t}} n_{\mathrm{r}}}\right\}\right\}\right|^{2},
$$

where $X Y=\{\mathrm{VV}, \mathrm{VH}, \mathrm{HV}, \mathrm{HH}\}$ indicates the polarized channel, and the Hann window is multiplied with CTF then the inverse Fourier transform is performed. To minimize the effect of noise, we can average the CTF over all good snapshots [5] then calculate the PDP. From the PDP, the reverberation time can be further calculated. [6] The reverberation time of an indoor channel is directly related to the PDP's exponential slope stemming from the multiple scattering in a closed environment. Fixed delay range or fixed power range is applied to PDP to calculate the reverberation time, and the range is usually visually determined by taking into account the effects of noise floor $[6,7]$. The double-directional PAS at frequency point $f_{k}$ is

$\operatorname{PAS}\left(\phi_{\mathrm{R}}, \theta_{\mathrm{R}}, \phi_{\mathrm{T}}, \theta_{\mathrm{T}}, f_{k}\right)=\left|\boldsymbol{A}_{\mathrm{R}}^{*}\left(\phi_{\mathrm{R}}, \theta_{\mathrm{R}}\right) \boldsymbol{H}\left(f_{k}\right) \boldsymbol{A}_{\mathrm{T}}^{H}\left(\phi_{\mathrm{T}}, \theta_{\mathrm{T}}\right)\right|^{2}$,

where $\boldsymbol{A}_{\mathrm{R}}\left(\phi_{\mathrm{R}}, \theta_{\mathrm{R}}\right) \in \mathbb{C}^{2 \times 16}$ is the radiation pattern of $\mathrm{Rx}$ at direction $\left(\phi_{\mathrm{R}}, \theta_{\mathrm{R}}\right), \boldsymbol{A}_{\mathrm{T}}\left(\phi_{\mathrm{T}}, \theta_{\mathrm{T}}\right) \in \mathbb{C}^{2 \times 16}$ is the radiation pattern of $\mathrm{Tx}$ at direction $\left(\phi_{\mathrm{T}}, \theta_{\mathrm{T}}\right), \boldsymbol{H}\left(f_{k}\right)=$ $\left[\boldsymbol{H}_{\mathrm{VV}}\left(f_{k}\right), \boldsymbol{H}_{\mathrm{VH}}\left(f_{k}\right) ; \boldsymbol{H}_{\mathrm{HV}}\left(f_{k}\right), \boldsymbol{H}_{\mathrm{HH}}\left(f_{k}\right)\right] \in \mathbb{C}^{16 \times 16} .\{\cdot\}^{*}$ indicate the vector/matrix conjugate, and $\{\cdot\}^{H}$ indicate the conjugate transpose. In addition, CLEAN algorithm [5], a low complexity iterative algorithm, is used to extract the delay, the angle of arrival (AoA), the angle of departure (AoD) and the complex polarimetric gain of multipath components from CTF and array patterns. It is a grid search based algorithm and the accuracy is linked to the grid size. Wideband CTF modeled by plane waves is given as

$\boldsymbol{H}\left(f_{k}\right)=\sum_{l} \exp \left(-j 2 \pi f_{k} \tau_{l}\right) \boldsymbol{A}_{\mathrm{R}}^{T}\left(\phi_{\mathrm{R}, l}, \theta_{\mathrm{R}, l}\right) \boldsymbol{\alpha}_{l} \boldsymbol{A}_{\mathrm{T}}\left(\phi_{\mathrm{T}, l}, \theta_{\mathrm{T}, l}\right)$.

Assuming the multipath components are resolvable, the joint maximum likelihood estimate of the directions cor- 
responding to the strongest multipath is given by

$$
\begin{aligned}
& \left(\hat{\tau}_{l}, \hat{\phi}_{\mathrm{T}, l}, \hat{\theta}_{\mathrm{T}, l}, \hat{\phi}_{\mathrm{R}, l}, \hat{\theta}_{\mathrm{R}, l}\right)
\end{aligned}
$$

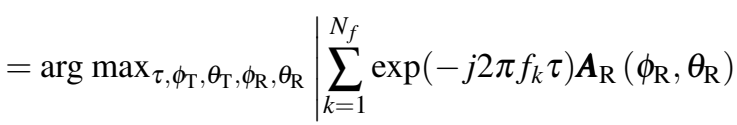

$$
\begin{aligned}
& \boldsymbol{H}^{*}\left(f_{k}\right) \boldsymbol{A}_{\mathrm{T}}^{T}\left(\phi_{\mathrm{T}}, \theta_{\mathrm{T}}\right) \mid .
\end{aligned}
$$

The estimated path gain of the corresponding component is the same as in [5]. At one time, only one path is estimated, then is subtracted from the current CTF. CLEAN algorithm is also applicable for narrowband channel.

\section{Numerical Examples}

Figure 4 shows the examples of PDP of measured channels. From the figure, it is obvious that the channels when person stands at $\mathrm{P} 4$ and $\mathrm{P} 2$, which are locations outside of the 1st Fresnel zone between Tx and Rx, have similar PDP with that of the background channel without person. The channels when there are two persons located inside the 1st Fresnel zone, has a more steep PDP "tail" than that when there is only one person inside. The slope of the PDP "tail" is described by the term of reverberation time, where the values of all locations are summarized in Table 2 . The observations are as follows. First, no matter with the presence of 1 person or 4 persons, when no person stands inside the 1st Fresnel zone, the reverberation time is similar as that of the background channel. Second, when one person stands inside the 1st Fresnel zone, the reverberation time tends to be around 7 ns lower than that of the background channel. Third, when two persons stand inside the 1st Fresnel zone, the reverberation time tends to be more than 12 ns lower than that of the background channel; in this case, the values when person is in FO posture tend to be larger than that when person is in FP posture. The observations from the measured data provide us hints in the perspective of detecting persons utilizing channel properties. The deployment of link ends should consider the 1st Fresnel zone and cover as much area as possible in room. The number of persons can be estimated from the variation of the reverberation time of current channel comparing to that of the background channel. The variations on reverberation time depend on the posture of persons' figures to the link's direct line.

It is worth mentioning that the array pattern is only available at center frequency for the moment, thus the PAS is calculated at center frequency. For this reason, the narrowband version of CLEAN algorithm is used, where the angular grid size is 1 degree. Fig. 5 show two examples of extracted paths seen from different cut planes at Tx and Rx sides, where the length of each path is proportional to the path gain in dB. From Fig. 5 (b) and (d), the new paths at around $y_{t}=0$ and $y_{r}=0$ are obvious due to the person. Most paths are effected in the path gain due to the "breakin" of person. However, any path estimation algorithm has errors; even through it is intuitive to observe the dead/new, affected, unaffected paths, those observations could be due

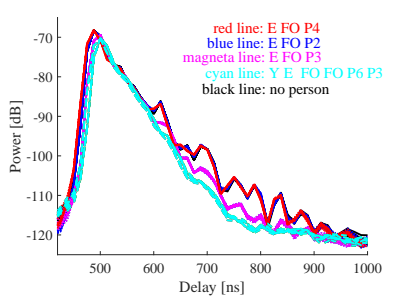

(a)

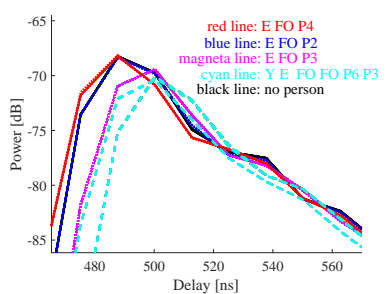

(b)
Figure 4. PDP of the good snapshots when person $\mathrm{E}$ stands at $\mathrm{P} 2, \mathrm{P} 3, \mathrm{P} 4$, when person $(\mathrm{Y}, \mathrm{E})$ stand at $(\mathrm{P} 6, \mathrm{P} 3)$, and when no person

Table 2. Reverberation Time Calculated from PDP

\begin{tabular}{cccc}
\hline \multicolumn{2}{c}{$\begin{array}{c}\text { Number of person } \\
\text { inside 1st } \\
\text { in room } \\
\text { Fresnel zone }\end{array}$} & $\begin{array}{c}\text { Reverberation } \\
\text { time when FO, } \\
\text { median values }\end{array}$ & $\begin{array}{c}\text { Reverberation } \\
\text { time when FP, } \\
\text { median values }\end{array}$ \\
\hline 0 & 0 & \multicolumn{2}{c}{$46.9 \mathrm{~ns}$} \\
\hline \multirow{2}{*}{1} & 0 & $47 \mathrm{~ns}$ & $47 \mathrm{~ns}$ \\
& 1 & $39.8 \mathrm{~ns}$ & $40 \mathrm{~ns}$ \\
\hline \multirow{2}{*}{2} & 0 & $47.3 \mathrm{~ns}$ & $47.6 \mathrm{~ns}$ \\
& 1 & $39.3 \mathrm{~ns}$ & $40.4 \mathrm{~ns}$ \\
& 2 & $34.4 \mathrm{~ns}$ & $27.8 \mathrm{~ns}$ \\
\hline \multirow{2}{*}{3} & 0 & $46.8 \mathrm{~ns}$ & $47.5 \mathrm{~ns}$ \\
& 1 & $40.7 \mathrm{~ns}$ & $40.5 \mathrm{~ns}$ \\
& 2 & $32.1 \mathrm{~ns}$ & $29.6 \mathrm{~ns}$ \\
\hline \multirow{2}{*}{4} & 0 & $47.6 \mathrm{~ns}$ & $48.9 \mathrm{~ns}$ \\
& 1 & $40.1 \mathrm{~ns}$ & $40 \mathrm{~ns}$ \\
& 2 & $34 \mathrm{~ns}$ & $30.9 \mathrm{~ns}$ \\
\hline
\end{tabular}

to the estimation errors. Hence, it is motivating to observe the PAS, where the errors could only come from the mismatch of array pattern used or the channel sounding error. Fig. 6 shows the PAS of background channel and channels with one person standing at P3, P4. Comparing (c) to (a), or (d) to (b), obvious differences can be observed. Those PAS variations are shown in Fig. 7 (a) and (b); it can be seen that except for the PAS variations at $x_{t}=0$ and $x_{r}=0$ where the person is supposed to be, there are other larger variations caused by the "break-in" of person. On the other hand, there is no obvious difference between Fig. 6 (e) and (a), or between (f) and (b); Fig. 7 (c) and (d) show the PAS variations. Although the values are very small, it also shows that the locations experiencing obvious PAS variations are multiple and are not necessarily to be the location of person.

\section{Discussions and Conclusion}

This paper explores the feasibility of indoor human positioning by using radio propagation channel properties of a single link. The PDP, reverberation time, PAS, extracted plane wave paths from the measured channel with the presence of person are compared to those from the background channel without person. The detection of person can be easily achieved through above comparisons. Particularly, the reverberation time calculated from the PDP tail can give 


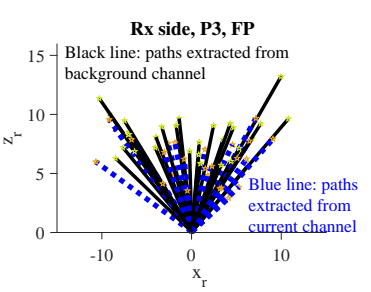

(a)

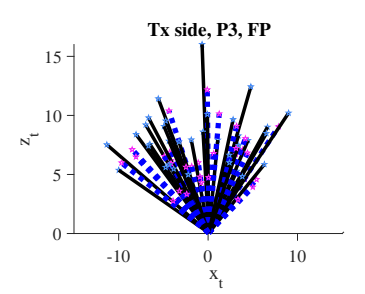

(c)

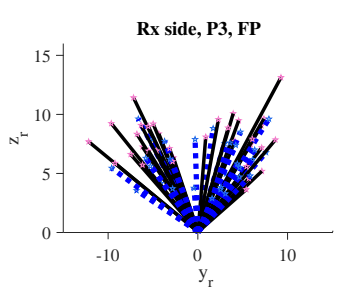

(b)

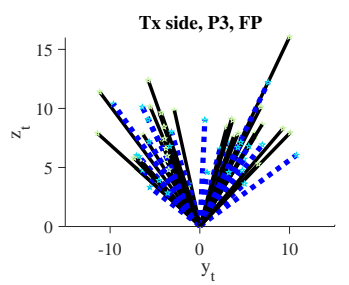

(d)
Figure 5. Extracted paths when person stands at P3 with FP (blue line) and that of background channel (black line)

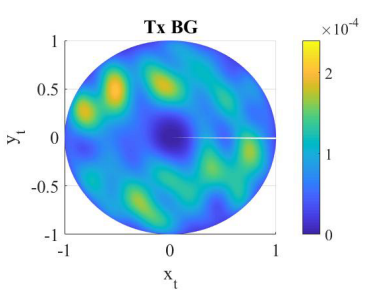

(a)

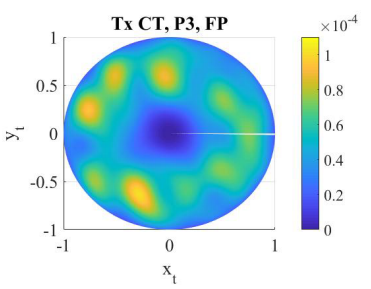

(c)

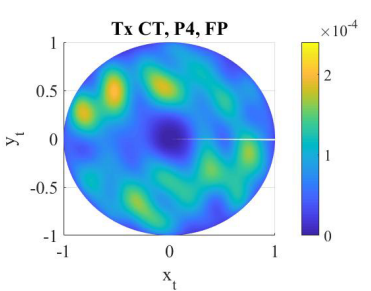

(e)

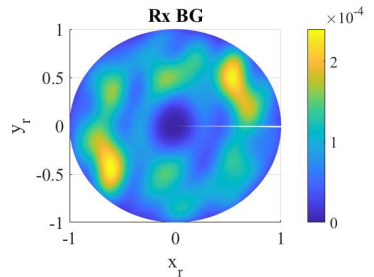

(b)

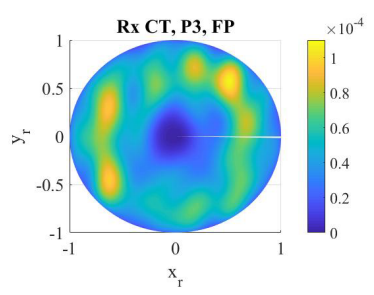

(d)

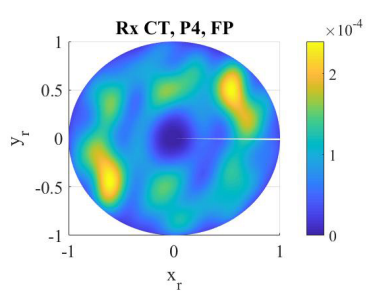

(f)
Figure 6. PAS, of background (BG) channel without person, and of current (CT) channel with person when E stands at P3 with FP, and that when Y stands at P4 with FP

estimations on how many persons located inside the 1st Fresnel zone of the link. For positioning of person, observing the changes of estimated paths and PAS alone can not give accurate location of person(s); the variations happen at multiple locations that could be around the person, or the second scatterers interacting with the waves scattered from human body. Further work will be the design of algorithms that differentiate the variations caused by person or

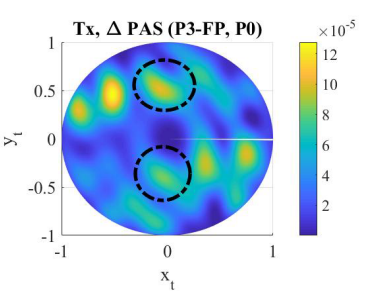

(a)

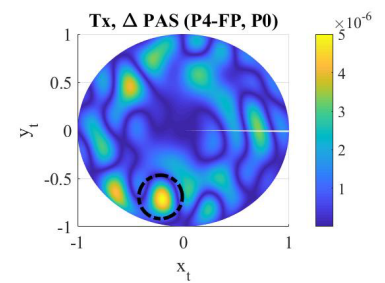

(c)

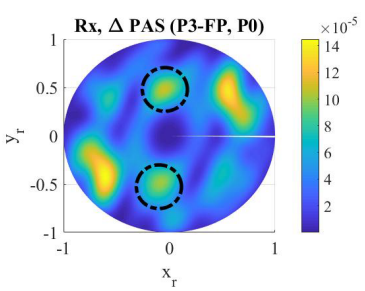

(b)

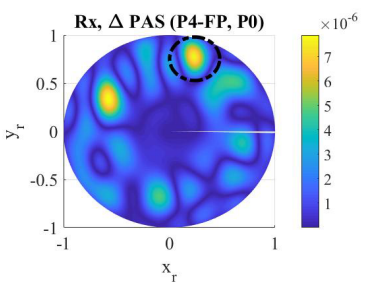

(d)
Figure 7. Variation between PAS of channel with person and that of background channel, where black circles indicate the PAS variations that are linked to person location

by second scatterers.

\section{References}

[1] N. Patwari, J. Wilson, "RF Sensor Networks for Device-Free Localization: Measurements, Models and Algorithms," Proceedings of the IEEE, vol. 98, no. 11, pp. 1961-1973, Jul. 2010.

[2] J. Shen, A.F. Molisch, "Discerning Direct and Indirect Paths: Principle and Application in Passive Target Positioning Systems," in Global Telecommunications Conference (GLOBECOM 2011), 2011 IEEE, Kathmandu, Nepal, Jan. 2012.

[3] J. Wilson, N. Patwari, "Radio Tomographic Imaging with Wireless Networks," IEEE Transactions on Mobile Computing, vol. 9, no. 5, pp. 621-632, May 2010.

[4] P. Laly, et al., "Real-time MIMO Channel Sounder Based on a Highly Flexible Software Architecture," in European Conference on Networks and Communications, Paris, France, Jun. 2015.

[5] V. Kristem, et al., "3D MIMO Outdoor-to-Indoor Propagation Channel Measurement," IEEE Trans. Wireless Commun., vol. 16, no. 7, Jul. 2017.

[6] G. Steinbock, et al., "Experimental Validation of the Reverberation Effect in Room Electromagnetics," IEEE Trans. Antennas Propag., vol. 63, no. 5, May 2015.

[7] A. Bamba, et al., "Experimental Assessment of Specific Absorption Rate Using Room Electromagnetics," IEEE Trans. Electromagn. Compat., vol. 54, no. 4, Aug. 2012. 